\title{
Laboratory Investigation of Preclinical Visual-Quality Metrics and Halo-Size in Enhanced Monofocal Intraocular Lenses
}

\author{
Grzegorz Łabuz · Hyeck-Soo Son · Tadas Naujokaitis · Timur M. Yildirim • \\ Ramin Khoramnia · Gerd U. Auffarth (D)
}

Received: September 6, 2021 / Accepted: October 8, 2021 / Published online: October 24, 2021

(c) The Author(s) 2021

\begin{abstract}
Introduction: This study aims to compare preclinical visual-quality metrics and halo size of intraocular lenses (IOL) with enhanced intermediate vision to a standard monofocal lens.

Methods: Three monofocal- IOL models with an extended-depth-of-focus (EDoF) intended for monocular implantation (Tecnis ICB00, AE2UV/ZOE, and IsoPure) and one for monovision (RayOne EMV) were compared against a standard monofocal lens (Tecnis ZCBO0). An optical-metrology station was used in the assessment of IOLs' optical quality in polychromatic light. The imaging quality was compared with metrics derived from the optical transfer function. Halo size was estimated from the projection of the point spread function under scotopic pupil.
\end{abstract}

Results: The monofocal IOL showed the highest image quality at the far focus. The ICB00's, the AE2UV/ZOE's, and the IsoPure's

Supplementary Information The online version contains supplementary material available at https:// doi.org/10.1007/s40123-021-00411-9.

G. Łabuz · H.-S. Son · T. Naujokaitis ·

T. M. Yildirim · R. Khoramnia · G. U. Auffarth $(\bowtie)$

The David J Apple Center for Vision Research,

Department of Ophthalmology, University Hospital

Heidelberg, Im Neuenheimer Feld 400, 69120

Heidelberg, Germany

e-mail: Gerd.Auffarth@med.uni-heidelberg.de performance at - 1D was superior to that of the monofocal lens. The monocular defocus tolerance of the RayOne EMV was comparable with that of the ZCB00. The RayOne EMV's intermediate range was improved in a monovision configuration ( $-1 \mathrm{D}$ offset). This approach, however, yielded the largest halo area, i.e., 53\% of the ZCB00's halo, compared to $34 \%$ for the IsoPure, $14 \%$ for the AE2UV/ZOE, and $8 \%$ for the ICB00.

Conclusion: The mono-EDoF models have a clear advantage over the standard monofocal lens by expanded imaging capability beyond $-0.5 \mathrm{D}$. Although the RayOne EMV provided the largest (binocular) visual-range extension, it was at the expense of monocular vision and higher susceptibility to halo. The ICBOO's and the AE2UV/ZOE's halo-profile was similar to that of the ZCBO0, indicating their low potential to induce photic phenomena.

Keywords: Enhanced monofocal; IOLs; MonoEDoF; MTF; Photic phenomena; Preclinical metrics; PTF 


\section{Key Summary Points}

Why carry out this study?

A new category of intraocular lenses (IOLs) has been introduced that improves intermediate vision of pseudophakic patients and induces a comparable level of photic phenomena to a standard monofocal implant. Given that those IOLs are of recent introduction, the scientific literature on their performance is scarce.

Quality metrics derived from the optical transfer function offer a high correlation with clinical visual acuity, which can be used to predict the clinical performance of new monofocal IOLs with an extended depth of focus prior to implantation.

\section{What was learned from the study?}

Enhanced-monofocal IOLs, based on a higher-order aspheric design, provide a comparable far optical quality and extended intermediate range while producing a monofocal-lens halo type. However, the depth-of-focus extension varies between the models, which should be taken into account in preoperative counseling.

Although the enhanced-monovision approach offered an extended visual range, a $-1 \mathrm{D}$ defocus and spherical aberration effects might increase a binocular perception of photic phenomena.

\section{INTRODUCTION}

Monofocal intraocular lenses (IOLs) with an enhanced intermediate function can provide an extended range of vision with a far-dominant performance, which, however, does not meet requirements for extended-depth-of-focus (EDoF) lenses set by the American Academy of
Ophthalmology [1]. A recent clinical trial assessing mono-EDoF technology demonstrated that patients with the Tecnis Eyhance ICBO0 have significantly better vision at intermediate distances but similar at far compared to those with a standard Tecnis lens [2]. In another clinical investigation, higher spectacle independence was observed at the intermediate range with the ICBOO compared to a monofocal lens [3]. The performance of the ICBO0 has also been documented in laboratory studies $[4,5]$. In those reports, however, only one IOL model has been evaluated. But with increasing recognition of this technology, various novel mono-EDoF lenses are being introduced, the precise characteristics of which have yet to be tested in the laboratory before they become routinely used in surgery.

Objective metrics derived from in vitro assessment of IOLs are widely used to evaluate the performance of new lens models [4-10] or failed implants $[11,12]$. One such metric is the optical transfer function (OTF) that represents the imaging ability of an optical system. This is a complex-valued function composed of the modulation transfer function (MTF) and the phase transfer function (PTF). The latest research indicates that optical-bench metrics offer a high correlation with clinical measurements of visual acuity (VA) and contrast sensitivity (CS) [7-9]. The optical quality of IOLs is typically assessed, during their manufacture, using the through-focus MTF, which is the internationally recognized standard for testing IOLs $[6,13]$. However, the inclusion of the PTF component may provide a more accurate estimate of the IOL's performance and its impact on postoperative vision [14].

Halos are dim circles of light surrounding a light source, which may occur due to refraction, diffraction, or ray aberration. In the case of IOLs, it often results from a simultaneous projection of multiple foci by an IOL. Thus, this postoperative complaint is typically associated with multifocal IOLs rather than monofocal ones $[15,16]$. Recent clinical and laboratory studies demonstrated a comparable amount of dysphotopsia with the ICBOO and the control monofocal Tecnis IOL [2-5], which may be used as a reference to test the susceptibility of new 
mono-EDoF designs to produce such phenomena.

This research aimed to apply the MTF and the PTF to compare the optical quality and the halo projection of the latest mono-EDoF models to that of a standard monofocal lens.

\section{METHODS}

\section{Intraocular Lenses}

In this laboratory investigation, we studied offthe-shelf IOLs; thus, ethics committee approval was not required. The following models were assessed.

- Tecnis Eyhance ICBO0 and Tecnis ZCB00 (both from Johnson \& Johnson Surgical Vision, Inc., Santa Ana, USA).

- AE2UV (Eyebright Medical Technology Inc, Beijing, China), distributed in Europe under the tradename ZOE Primus-HD (Ophthalmo Pro GmbH, Sankt Ingbert, Germany).

- IsoPure 1.2.3 (PhysIOL sa/nv, Liège, Belgium).

- RayOne EMV (Rayner Intraocular Lenses Limited, Worthing, UK).

Two IOL samples from each model were tested, each having the same refractive power of $+20 \mathrm{D}$.

The Tecnis ZCBOO and ICBO0 are made of the same hydrophobic acrylic material with a refractive index of 1.47 at $35^{\circ} \mathrm{C}$ and the Abbe number of 55 . The two models were designed to correct a spherical aberration (SA) of $0.27 \mu \mathrm{m}$ at $6 \mathrm{~mm}$ [17]. Despite a similar appearance, the Eyhance's anterior surface differs (by approximately 15\%) in its central, optical area from that of the ZCBOO-having an increase in lens power through utilizing higher-order aspheric components [4]. Thus, the Eyhance ICBO0 aims to enhance intermediate vision compared to the ZCB00 while maintaining identical correction of primary SA [4].

The AE2UV/ZOE is a hydrophobic-acrylic lens with a refractive index of 1.47 and the Abbe number of 57. The IOL features an aspheric design to lower the primary SA of the cornea by $-0.20 \mu \mathrm{m}$ at $6 \mathrm{~mm}$. A high-order aspheric surface enhances intermediate vision with increased SA aberration in the IOL center that gradually decreases towards the periphery. A smooth and continuous higher-order aspheric surface aims to minimize photic phenomena that may result from abrupt profile changes, such as observed in diffractive IOLs.

The IsoPure 1.2.3 is a hydrophobic-acrylic IOL with a refractive index of 1.52 and an Abbe number of 42 . The IsoPure features anterior and posterior aspheric surfaces with high-order aspheric terms to extend the visual range compared to a monofocal IOL while maintaining a good far-focus performance. A posterior surface of the lens has a conic profile to correct $-0.11 \mu \mathrm{m}$ of SA.

The RayOne EMV is made of (26\%) hydrophilic-acrylic material with a refractive index of 1.46 at $35^{\circ} \mathrm{C}$ and an Abbe number of 56. The RayOne EMV is offered as enhanced monovision IOL where a plano target is maintained in the dominant eye, and a power-offset is applied in the non-dominant eye. According to the manufacturer, a $1 \mathrm{D}$ offset offers a $2.25 \mathrm{D}$ depthof-focus extension in binocular vision. The inner part of the IOL includes positive SA to improve patients' distance vision in the nondominant eye.

\section{Optical-Metrology Analysis}

We used an OptiSpheric IOL PRO2 (Trioptics GmbH, Wedel, Germany) device in the assessment of the optical performance of the study IOLs. This setup was built based on the ISO standard configuration that includes a corneal model with $0.28 \mu \mathrm{m}$ of SA at $5.15 \mathrm{~mm}$ [13]. The device's spectral property mimics the spectral sensitivity of the human eye with a peak intensity of $555 \mathrm{~nm}$. The OTF components (i.e., the MTF and the PTF) were derived from the line spread function projected by the device through the tested IOL [18]. The longitudinal chromatic aberration of the model eye (without an IOL) was approximately 1D between 480 and $644 \mathrm{~nm}$ [19]. We assessed the optical quality parameters for the aperture size of $3 \mathrm{~mm}$ and $4.5 \mathrm{~mm}$ at the IOL plane [20]. After finding the best focus using the MTF criterion, the IOL's 
tolerance to defocus was tested within $\mathrm{a}+0.50 \mathrm{D}$ to $-2.50 \mathrm{D}$ range (0.25-D resolution). At each focus-point, sagittal and tangential MTFs and PTFs were acquired, and the two meridians were averaged. Besides, at the $3-\mathrm{mm}$ pupil, we recorded the 1951 USAF resolution test chart.

The weighted optical transfer function (wOTF) was calculated as described by Alarcon et al. [7], who in a later study also applied this metric to evaluate the optical quality of the ICB00 lens [4]. The wOTF was derived from the measured MTF and PTF at $3 \mathrm{~mm}$ and weighted by the neural CS function (CSF) using the following formula:

${ }_{\mathrm{wOTF}}=\frac{d}{150} \sum_{f=1}^{\frac{150}{d}} \operatorname{MTF}(f d) \cos (\operatorname{PTF}(f d)) \operatorname{CSF}(f d)_{\text {neural }}$,

where $f$ is the spatial frequency expressed in $\mathrm{lp} /$ $\mathrm{mm}$ with a resolution of $d=1 \mathrm{lp} / \mathrm{mm}$. The neural CSF data were obtained from the work of Campbell and Green [21]. The wOTFb change with defocus (where $b$ is -0.36) has been reported to correlate strongly with clinically measured VA, and we compared this change between models $[7,22]$.

A polychromatic point spread function (PSF), i.e., an image of a $0.1-\mathrm{mm}$ pinhole, was used to assess the size of halos projected by the IOLs at the $4.5-\mathrm{mm}$ aperture $[4,16]$. A long transformation of each recorded image was applied, and the background noise was removed. The halo size was defined as the total area (2-D images) with the assumption of circularity.

\section{Binocular Summation}

Given that the RayOne EMV is implanted bilaterally through monovision, we performed the binocular summation of recorded USAFchart and PSF images. We applied a quadratic summation proposed by Legge and Rubin for a pair of images corresponding to the left and right eye with the defocus offset of $1 \mathrm{D}[23,24]$.

\section{Data Analysis}

The analysis of optical-quality data and images were performed with custom-made software developed in MATLAB (MathWorks, USA).

\section{RESULTS}

The MTF curves of all IOL samples measured at the best focus through the $3-$ and $4.5-\mathrm{mm}$ apertures are presented in Fig. 1 with 2D MTF visualization shown in Fig. 1S (Supplementary Material).

At $3 \mathrm{~mm}$, the ZCBOO demonstrated the highest MTF values of the five models, particularly at higher spatial frequencies, with an average level of $0.50 \pm 0.00$ at $50 \mathrm{lp} / \mathrm{mm}$. The AE2UV/ZOE produced the average MTF @ 50 lp/ $\mathrm{mm}$ of $0.37 \pm 0.02$. At the same frequency, the ICB00's MTF was comparable, and that was $0.36 \pm 0.01$. The RayOne EMV's MTF was $0.40 \pm 0.00$, which was reduced dramatically after the $1 \mathrm{D}$ offset $(\mathrm{MTF}=0.04 \pm 0.01)$. The IsoPure's performance at $50 \mathrm{lp} / \mathrm{mm} \quad(\mathrm{MTF}=$ $0.41 \pm 0.01)$ was minimally better than found in the other mono-EDoF models.

At $4.5 \mathrm{~mm}$, the ZCBO0's high-frequency performance (MTF@50 lp/mm =0.39 \pm 0.01 ) was better than that of the mono-EDoF IOLs. The ICB00's MTF was $0.31 \pm 0.01$, comparable to that measured with the AE2UV/ZOE $(0.29 \pm 0.00)$ at $50 \mathrm{lp} / \mathrm{mm}$. For the IsoPure, it was $0.23 \pm 0.00$. The RayOne EMV's MTF at zero-defocus was $0.17 \pm 0.00$, but a simulation of $\mathrm{a}-1 \mathrm{D}$ refractive target resulted in a poor farfocus performance at $4.5 \mathrm{~mm}$ (MTF@50 lp/ $\mathrm{mm}=0.02 \pm 0.00$ ).

Figure 2 reports the $\mathrm{wOTF}^{\mathrm{b}}$ change with defocus for the five IOL models. The wOTF ${ }^{b}$ of the ZCB00 was minimally better at the best farfocus than that of the other models. However, at about $-0.5 \mathrm{D}$, the ICBO0, the AE2UV/ZOE, and the IsoPure demonstrated improved tolerance to defocus compared to the monofocal IOL. The RayOne EMV's and the ZCBO0's performance under defocus was comparable with the simulated plano refractive target. Although the $1 \mathrm{D}$ offset resulted in a worse $\mathrm{wOTF}^{\mathrm{b}}$ level of the RayOne EMV at the far focus, in binocular 

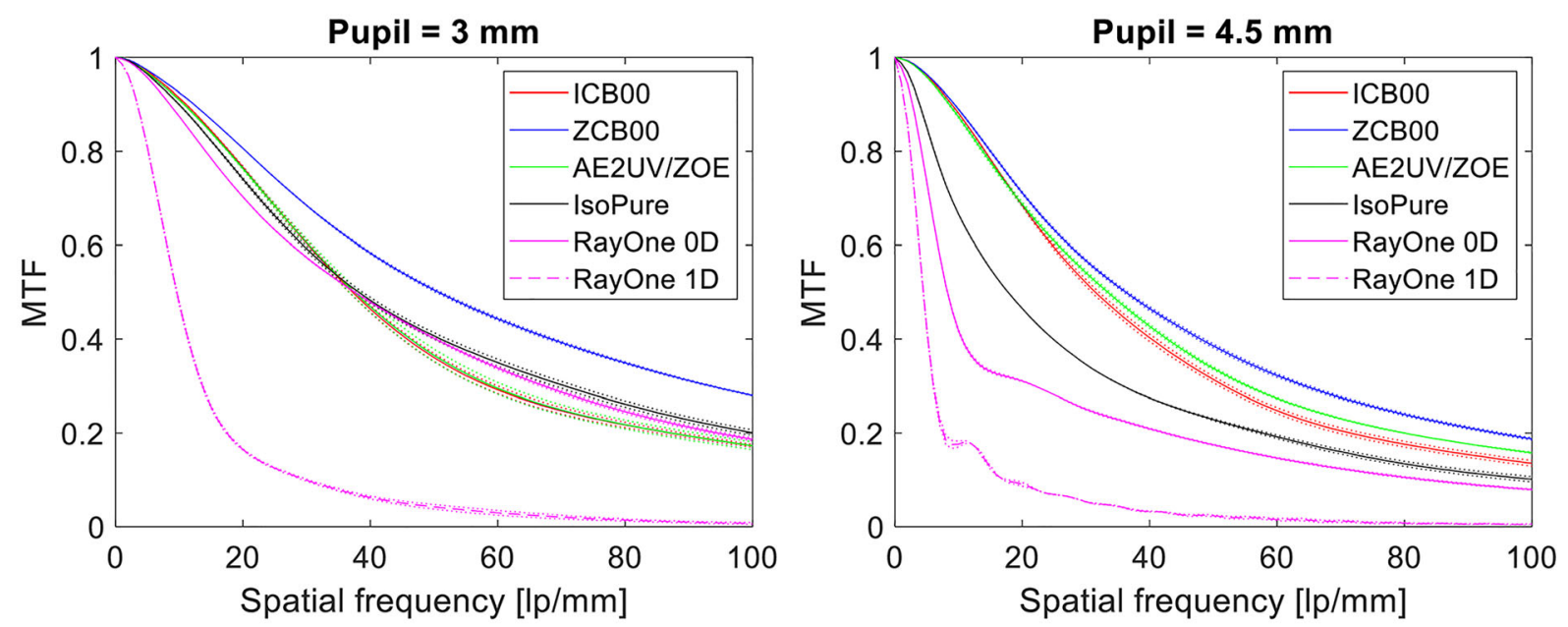

Fig. 1 Modulation transfer function (MTF) levels of the studied IOLs at the best focus for 3- and 4.5-mm apertures. The dotted lines show the values of each lens separately; the solid lines refer to the average of two samples

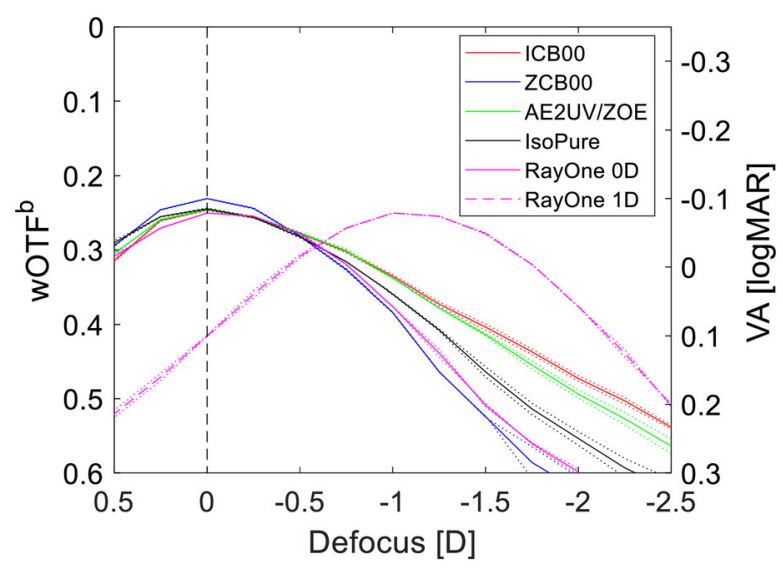

Fig. 2 Defocus tolerance of the weighted optical transfer function to the power of $b=-0.36\left(\right.$ wOTF $\left.^{\mathrm{b}}\right)$ assessed at $3 \mathrm{~mm}$ and visual acuity (VA) predictions presented on a secondary axis. The dotted lines show each lens' values separately; the solid lines refer to the average of two samples. The vertical dashed line indicates the position of the far focus

vision, the defocus tolerance may be further extended.

The resolution-test images confirm the wOTF $^{\mathrm{b}}$ results. Figure 3 presents the quadratic summation results for the RayOne EMV and the recorded (single) images for the other models. Figure 2S (Supplementary Material) shows the comparison between the USAF- chart photographs taken with the simulated plano and $-1 \mathrm{D}$ refractive target and the corresponding binocular image.

The PSF projection confirmed a similar halo profile among the $\mathrm{ZCBO0}$, the ICB00, and the AE2UV/ZOE (Fig. 4). The ZCBOO produced the halo radius of the smallest size $(1.73 \pm 0.06$ arcmin), which was closely followed by the ICBO0's $1.88 \pm 0.08$ arcmin and AE2UV/ZOE's $2.01 \pm 0.04$ arcmin. The IsoPure created a halo with an average size of $2.64 \pm 0.00$ arcmin. The RayOne EMV's binocular halo size was $3.67 \pm 0.18$ arcmin, which was the largest among the studied IOLs. Figure 3S (Supplementary Material) shows the PSF images of the RayOne EMV at zero, and - 1D defocus with the corresponding quadratic summation.

\section{DISCUSSION}

This study provides further evidence that mono-EDoF IOLs have the potential to improve the patient's visual function at the intermediate range while producing the optical performance that is close to that of standard monofocal IOLs at their best focus. Although the RayOne EMV showed a more extended range of vision than that of the other mono-EDoF models, the impact of binocular summation is challenging to predict in a laboratory setting. 

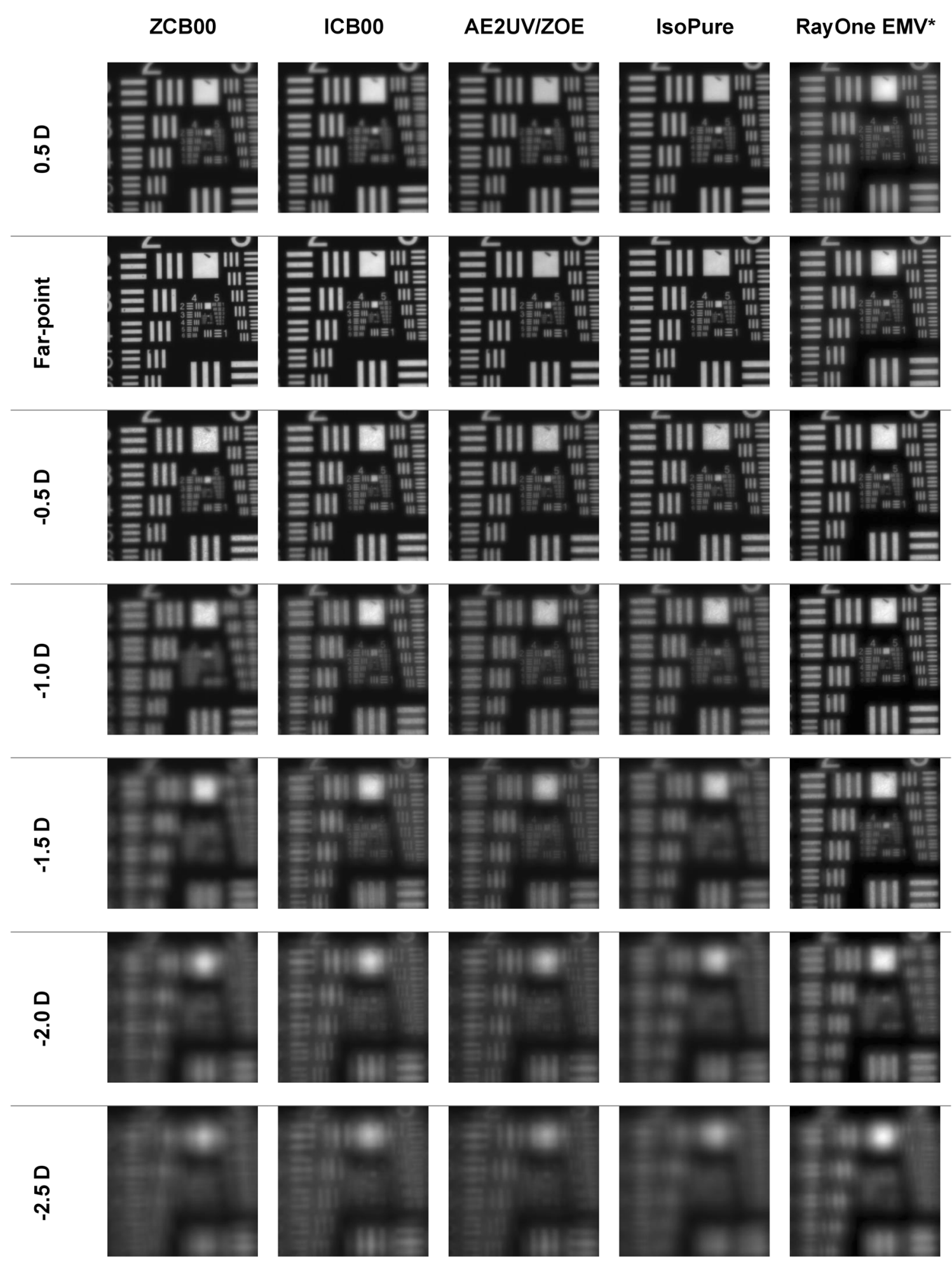

Fig. 3 US Air Force resolution targets recorded at a defocus range of $+0.5 \mathrm{D}$ to $-2.5 \mathrm{D}$ and the 3-mm aperture.

${ }^{*}$ Quadratic summation of two images with the defocus difference of $1 D$ 
Given that the studied IOLs are still relatively new to clinicians, we were not able to identify any peer-reviewed publication on the in vivo or in vitro functioning of the AE2UV/ZOE, IsoPure, and the RayOne EMV. This gap underlines the importance of our research. In a recent publication, Mencucci et al. made a clinical comparison of the ZCBOO and ICBOO [3]. Various aspects of the visual function were assessed, including VA, CS, and the perception of photic phenomena. They found that monocular corrected distance VA differs only by 0.01 in favor of the ZCBO0 group. However, monocular distance corrected intermediate VA measured at $66 \mathrm{~cm}(\sim 1.5 \mathrm{D})$ was improved in patients with the mono-EDoF lens by one line [3]. Those findings are in agreement with the results of our in vitro evaluation, as we also found that ZCBOO had a minimally higher wOTF ${ }^{\mathrm{b}}$ than the ICB00 at far-point, but worse at intermediate by 0.12 . Following the conversion of the $\mathrm{wOTF}^{\mathrm{b}}$ to logMAR VA using a model proposed by Alarcon et al. [7, 22], a 1.3 line difference between the two Tecnis IOLs can be predicted at $-1.5 \mathrm{D}$.

In a paper by Auffarth et al., the European multicenter study results on the comparison between the ICBOO and ZCBOO IOLs were presented [2]. They found that the mean corrected distance VA was $-0.02 \pm 0.01 \log$ MAR in the mono-EDoF group and $-0.06 \pm 0.01 \log$ MAR in the standard-monofocal one. In the current in vitro study, we also found a minimally decreased $\mathrm{WOTF}^{\mathrm{b}}$ of the ICBOO IOLs at the far focus with the estimated VA effect of $0.02 \mathrm{log}$ MAR in favor of the ZCBOO group. At the intermediate range $(66 \mathrm{~cm})$, the distance corrected VA was $0.19 \pm 0.02 \log$ MAR and $0.31 \pm 0.02 \log$ MAR in the mono-EDoF and monofocal patients, respectively. They reported the mean improvement of $0.11 \pm 0.02 \log \mathrm{MAR}$, which is close to the predicted level from our in vitro assessment. At $1.5 \mathrm{D}$ defocus, the AE2UV/ZOE's wOTF ${ }^{\mathrm{b}}$ only slightly differed from that of the ICBO0, which would result in a minimally lower VA by $0.01 \log$ MAR and 0.03 $\log$ MAR at $2.5 \mathrm{D}$ according to the model $[7,22]$. The AE2UV/ZOE provides a clear advantage over the standard monofocal lens, as at $66 \mathrm{~cm}$ and $40 \mathrm{~cm}$, one line VA improvement $(0.1 \mathrm{log}$ MAR) is expected at both distances compared to the ZCB00. For the IsoPure it is less, about 0.07 $\log$ MAR at $1.5 \mathrm{D}$ of defocus. Although RayOne EMV showed $<0.02 \log$ MAR difference over the monofocal lens in the 'dominant eye' simulation, in the 'non-dominant eye' a nearly 3-line (0.27 logMAR) improvement would be expected. However, these laboratory results should be confirmed in a clinical study.

The ICBOO and the ZCBOO have also been compared in a laboratory setting. Alarcon et al. studied their optical quality by measuring the MTF and the OTF in polychromatic light using their optical bench [4]. Despite expected differences in our and the J\&J Vision group's optical setup, they also predicted a $0.12 \log$ MAR improvement at the intermediate range after the implantation of their mono-EDoF lens compared to the standard monofocal model. Furthermore, Alarcon and associates reported a negligible impact of higher-order aberrations and pupil size on their comparison. However, Vega et al. found a shift of the through-focus MTF area by $-0.50 \mathrm{D}$ at $2 \mathrm{~mm}$, indicating some level of pupil dependency [5]. Although Auffarth et al. did not see clinically significant changes for pupils larger than $2.5 \mathrm{~mm}$ [2], the comparison for smaller pupils was not performed due to insufficient sample size. More research is needed to determine the impact of pupil size on the visual quality of patients with the new mono-EDoF IOLs.

The European multicenter clinical study concluded that the occurrence of photic phenomena is comparable between the ICBOO and ZCB00 IOLs [2]. Mencucci et al. also reported insignificant differences between the two groups in the perception of glare and halo [3]. The two laboratory studies have shown that the ICBOO and the ZCBOO produce a similar halo pattern with a slightly lower intensity profile for the latter model $[4,5]$. Our analysis of the PSF may further reinforce those findings, as we also reported that the observed halo was only $8 \%$ larger in the ICBO0 than that recorded through the monofocal lens. This difference was slightly increased but still comparable for the AE2UV/ ZOE, which was $14 \%$. Our results indicate that the AE2UV/ZOE's contribution to photic phenomena is minimal, and it is comparable to that of a monofocal lens. 


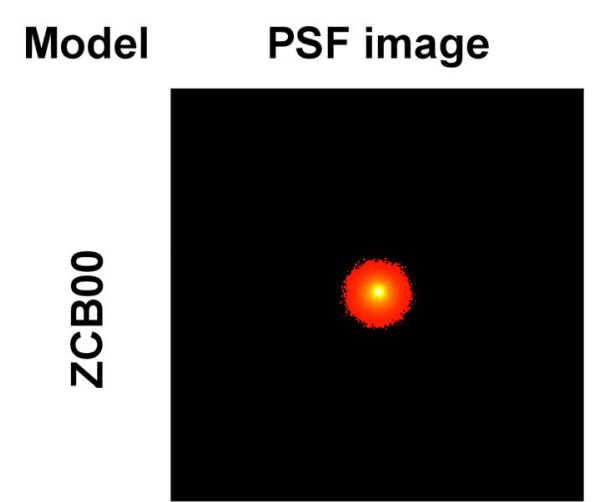

PSF cross-section
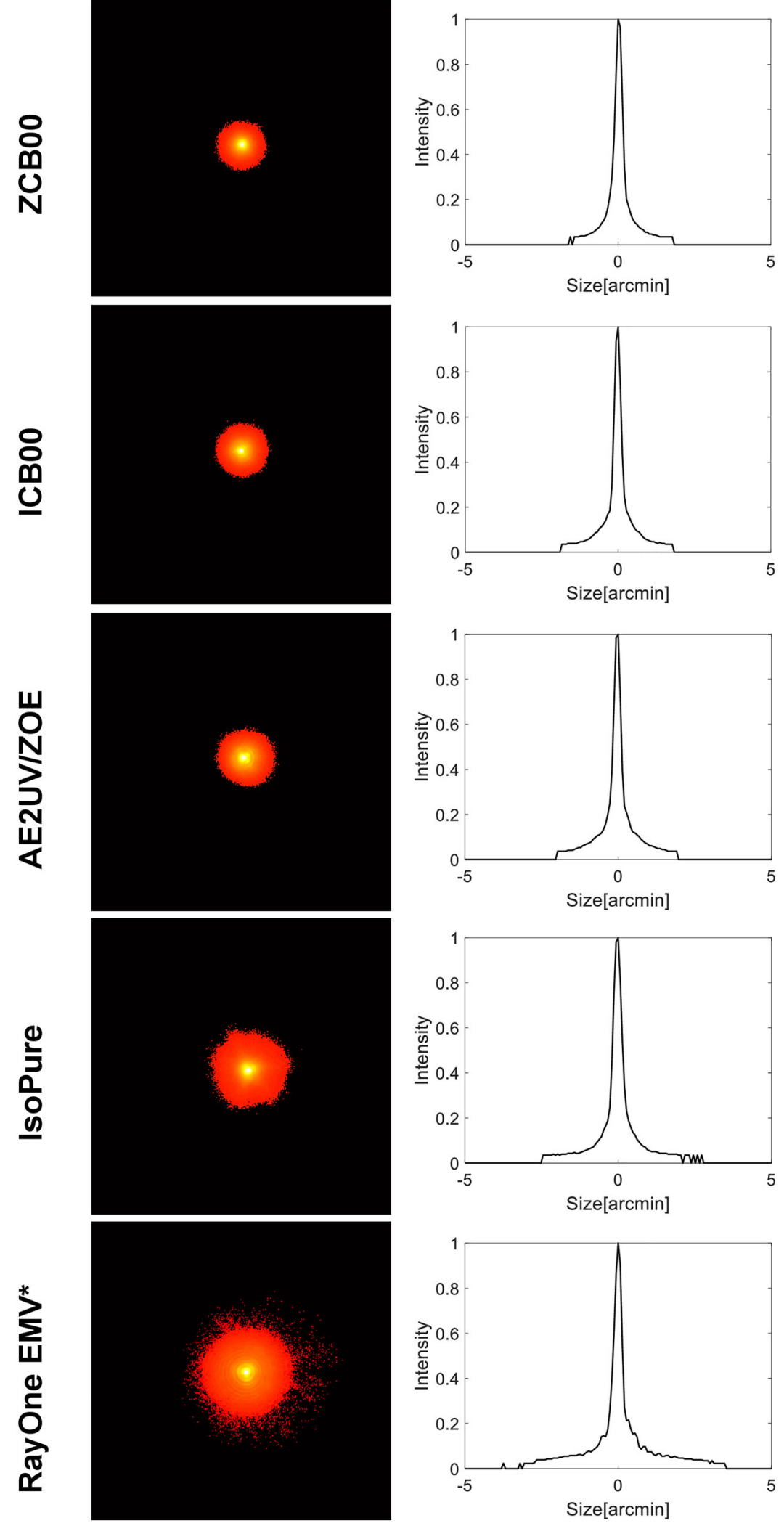
4Fig. 4 Logarithmic images of the recorded polychromatic point spread function (PSF, left panels) at $4.5 \mathrm{~mm}$ with color-coding. The right panels present the linear crosssection of the PSF's intensity profile. *Quadratic summation of two images with the defocus difference of $1 D$

The IsoPure and the RayOne EMV produced a halo pattern that was larger than that of the ZCB00 by $34 \%$ and $53 \%$, respectively. One potential explanation of this observation is a lower SA correction. In the current study, the cornea model exerts $0.28 \mu \mathrm{m}$ of SA while the IsoPure corrects $-0.11 \mu \mathrm{m}$, but the RayOne EMV is considered 'aberration neutral.' Although the choice of this SA correction might have been motivated by the broad range of SA distribution in the population, in this in vitro model, we simulated the average SA value of the cornea [17], which favors the lens design of the Tecnis IOLs. In such a setting, however, the IsoPure and RayOne EMV performance at the scotopic pupil could be compromised, which might also result in an increased halo-pattern due to a higher level of SA. Two other factors may also contribute to the increased halo size in the RayOne EMV.

1. The Rayner model induces additional positive SA to optimize the far vision of the non-dominant eye. Thus, we expect that increased SA effects may spread the RayOne EMV's halo-profile and reduce the optical quality, as shown in Fig. 1.

2. Similarly, defocus blur also has the potential to increase the halo perception $[25,26]$.

Figure 4 shows the quadratic summation of the focus and unfocused (1D offset) PSF. Although both log images (Fig. 3S, Supplementary Material) show an extended halo pattern around the central disk, defocus appears to produce higher-intensity effects in the simulation of the 1-D offset. Johannsdottir and Stelmach found that subjective complaints about photic phenomena under low-light conditions are more common in monovision compared to binocular emmetropia [25]. Still, the monovision approach provides fewer halo symptoms than the implantation of a diffractive-bifocal
IOL, as Zhang et al. have demonstrated [26]. Thus, whether the observed increased halo profile in the RayOne EMV may induce functionally significant photic phenomena is a question that must be addressed in a clinical study.

In a healthy emmetropic eye, the summation of the visual information from both eyes results in enhanced visual perception compared to monocular vision. Campbell and Green estimated that binocular summation improves the visual quality by a factor of $\sqrt{2}$ [27]. However, a recent meta-analysis by Baker and Lygo indicates that the ratio between binocular and monocular perception may be higher, implying a multifactorial mechanism underlying this process [28]. Conversely, the binocular summation may also be hampered, e.g., under monovision correction, with the ratio close to or less than 1 indicating the deterioration of binocular vision $[29,30]$. If we assume that the binocular summation is 1 for the RayOne EMV and $\sqrt{2}$ for other IOLs, the far-focus wOTF $^{\mathrm{b}}$ increases for all models but one (Fig. 5). The binocular summation may also minimize the image quality difference between the RayOne EMV and the other mono-EDoF models at the intermediate distance, which may be a good

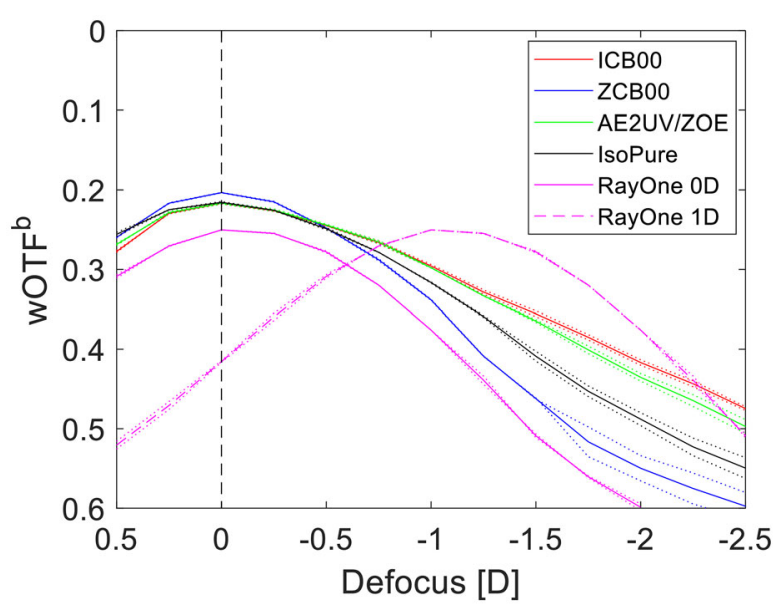

Fig. 5 Simulations of WOTF $^{\mathrm{b}}$ binocular summation at $3 \mathrm{~mm}$ and under defocus. The dotted lines show each lens' values separately; the solid lines refer to the average of two samples. The vertical dashed line indicates the position of the far focus 
option for patients who may not tolerate well dissimilarity in the blur perception between the eyes. More research is needed to determine the impact of enhanced monovision on patients' binocular performance.

The use of one pupil size (i.e., $3 \mathrm{~mm}$ ) might not fully represent a clinical situation [23], which may be considered a limitation of the current study. However, the wOTF metric was computed by Alarcon et al. from laboratory data obtained at $3 \mathrm{~mm}$ [7]; thus, its implementation for other pupil sizes requires validation. This should be a subject of further research.

\section{CONCLUSIONS}

We found that the mono-EDoF models' farpoint MTF was minimally lower than that of the standard monofocal IOL, but the expected impact on clinical VA is negligible. All monoEDoF models have the potential to extend the patient's intermediate vision beyond the range of a standard monofocal lens. Our laboratory results for the two Tecnis IOLs appear to conform to the published results from other researchers. We found that the AE2UV/ZOE and the ICBO0 produced a halo pattern comparable to that of the ZCB00 indicating a low potential of these IOLs to induce photic phenomena.

\section{ACKNOWLEDGEMENTS}

Donald J. Munro contributed to the review of the manuscript. Ethics committee approval was not required for this laboratory study.

Funding. This work was supported by unrestricted research grants from Klaus Tschira Stiftung and Eyebright Medical Technology Co., Ltd. The sponsors had no role in the design, execution, interpretation, or writing of the study. T. M. Yildirim is funded by the PhysicianScientist Program of the Heidelberg University, Faculty of Medicine, Heidelberg, Germany.

Authorship. All named authors meet the International Committee of Medical Journal Editors (ICMJE) criteria for authorship for this article, take responsibility for the integrity of the work as a whole, and have given their approval for this version to be published.

Authorship Contributions. Conceptualization, GE and GUA; methodology, GL; investigation, GE, H-SS, TN, TMY, RK and GUA; data analysis, GE, H-SS, TN, and TMY; resources, GUA and RK; draft preparation, GŁ and H-SS; review and editing, GE, H-SS, TN, TMY, RK and GUA; supervision, GE, RK and GUA; project administration, GUA; funding acquisition, GUA. All authors have read and agreed to the published version of the manuscript.

Disclosures. Gerd Uwe Auffarth reports grants, personal fees, non-financial support and consulting fees from Johnson \& Johnson and Alcon, grants, personal fees, and non-financial support from Carl Zeiss Meditec, Hoya, Kowa, Oculentis/Teleon, Rayner, Santen, Sifi, Ursapharm, grants and personal fees from Biotech, Oculus, EyeYon grants from Acufocus, Contamac, Glaukos, Physiol, Rheacell, outside the submitted work. Ramin Khoramnia reports grants, personal fees, and non-financial support from Alcon, Johnson\&Johnson, Hoya, Physiol, Rayner, personal fees, and non-financial support from Kowa, Ophtec, Oculentis/Teleon, Santen, and Acufocus, outside the submitted work. Grzegorz Łabuz, Hyek-Soo Son, Tadas Naujokaitis, and Timur M. Yildirim have nothing to disclose.

Data Availability. The datasets generated during and/or analyzed during the current study are available from the corresponding author on reasonable request.

Open Access. This article is licensed under a Creative Commons Attribution-NonCommercial 4.0 International License, which permits any non-commercial use, sharing, adaptation, distribution and reproduction in any medium or format, as long as you give appropriate credit to the original author(s) and the source, provide a link to the Creative Commons licence, and indicate if changes were made. The images or other third party material in this article are included in the article's Creative Commons 
licence, unless indicated otherwise in a credit line to the material. If material is not included in the article's Creative Commons licence and your intended use is not permitted by statutory regulation or exceeds the permitted use, you will need to obtain permission directly from the copyright holder. To view a copy of this licence, visit http://creativecommons.org/licenses/by$\mathrm{nc} / 4.0 /$.

\section{REFERENCES}

1. MacRae S, Holladay JT, Glasser A, Calogero D, Hilmantel G, Masket S, et al. Special report: American Academy of Ophthalmology Task Force consensus statement for extended depth of focus intraocular lenses. Ophthalmology. 2017;124(1):139-41.

2. Auffarth GU, Gerl M, Tsai L, Janakiraman DP, Jackson B, Alarcon A, Dick HB. Quantum Study Group. Clinical evaluation of a new monofocal IOL with enhanced intermediate function in patients with cataract. J Cataract Refract Surg. 2021;47(2): 184-91.

3. Mencucci R, Cennamo M, Venturi D, Vignapiano R, Favuzza E. Visual outcome, optical quality, and patient satisfaction with a new monofocal IOL, enhanced for intermediate vision: preliminary results. J Cataract Refract Surg. 2020;46(3):378-87.

4. Alarcon A, Cánovas C, Koopman B, Weeber H, Auffarth GU, Piers PA. Enhancing the intermediate vision of monofocal intraocular lenses using a higher order aspheric optic. J Refract Surg. 2020;36(8):520-7.

5. Vega F, Millán MS, Gil MA, Garzón N. Optical performance of a monofocal intraocular lens designed to extend depth of focus. J Refract Surg. 2020;36(9): 625-32.

6. Norrby NS, Grossman LW, Geraghty EP, Kreiner CF, Mihori M, Patel AS, et al. Determining the imaging quality of intraocular lenses. J Cataract Refract Surg. 1998;24(5):703-14.

7. Alarcon A, Canovas C, Rosen R, Weeber H, Tsai L, Hileman $\mathrm{K}$, et al. Preclinical metrics to predict through-focus visual acuity for pseudophakic patients. Biomed Opt Express. 2016;7(5):1877-88.

8. Vega F, Millán MS, Garzón N, Altemir I, Poyales F, Larrosa JM. Visual acuity of pseudophakic patients predicted from in-vitro measurements of intraocular lenses with different design. Biomed Opt Expr. 2018;9(10):4893-906.

9. Łabuz G, Auffarth GU, Özen A, van den Berg TJ, Yildirim TM, Son H-S, et al. The effect of a spectral filter on visual quality in patients with an extendeddepth-of-focus intraocular lens. Am J Ophthalmol. 2019;208:56-63.

10. Fernández J, Rodríguez-Vallejo M, Martínez J, Burguera N, Piñero DP. Prediction of visual acuity and contrast sensitivity from optical simulations with multifocal intraocular lenses. J Refract Surg. 2019;35(12):789-95.

11. Łabuz G, Yildirim TM, van den Berg TJ, Khoramnia R, Auffarth GU. Assessment of straylight and the modulation transfer function of intraocular lenses with centrally localized opacification associated with the intraocular injection of gas. J Cataract Refract Surg. 2018;44(5):615-22.

12. Yildirim TM, Labuz G, Khoramnia R, Son HS, Schickhardt SK, Lieberwirth I, et al. Impact of primary calcification in segmented refractive bifocal intraocular lenses on optical performance including straylight. J Refract Surg. 2020;36(1):20-7.

13. ISO-11979-2. Ophthalmic implants-intraocular lenses-part 2: optical properties and test methods. Geneva: The International Organization for Standardization; 2014.

14. Sarver EJ, Applegate RA. The importance of the phase transfer function to visual function and visual quality metrics. J Refract Surg. 2004;20(5): S504-7.

15. Schallhorn JM. Multifocal and extended depth of focus intraocular lenses: a comparison of data from the united states food and drug administration premarket approval trials. J Refract Surg. 2021;37(2):98-104.

16. Alba-Bueno F, Garzón N, Vega F, Poyales F, Millán MS. Patient-perceived and laboratory-measured halos associated with diffractive bifocal and trifocal intraocular lenses. Curr Eye Res. 2018;43(1):35-42.

17. Wang L, Dai E, Koch DD, Nathoo A. Optical aberrations of the human anterior cornea. J Cataract Refract Surg. 2003;29(8):1514-21.

18. Boreman GD. Modulation transfer function in optical and electro-optical systems. Bellingham: SPIE Press; 2001.

19. Łabuz G, Papadatou E, Khoramnia R, Auffarth GU. Longitudinal chromatic aberration and polychromatic image quality metrics of intraocular lenses. J Refract Surg. 2018;34(12):832-8. 
20. Koch DD, Samuelson SW, Haft EA, Merin LM. Pupillary and responsiveness: implications for selection of a bifocal intraocular lens. Ophthalmology. 1991;98(7):1030-5.

21. Campbell F, Green D. Optical and retinal factors affecting visual resolution. J Physiol. 1965;181(3): 576-93.

22. Heredia AA, Vidal CC, Rosén R, Weeber HA, Piers PA. Apparatus, systems and methods for improving visual outcomes for pseudophakic patients. Google Patents; 2017.

23. Legge GE, Rubin GS. Binocular interactions in suprathreshold contrast perception. Percept Psychophys. 1981;30(1):49-61.

24. Lee S, Carson D, Gu X, Breen M, Hong X, LempHull J, et al. Simulation of blended vision and monovision with multifocal intraocular lenses by image fusion. Investig Ophthalmol Vis Sci. 2019;60(9):3682.

25. Johannsdottir KR, Stelmach LB. Monovision: a review of the scientific literature. Optom Vis Sci. 2001;78(9):646-51.
26. Zhang F, Sugar A, Jacobsen G, Collins M. Visual function and patient satisfaction: comparison between bilateral diffractive multifocal intraocular lenses and monovision pseudophakia. J Cataract Refract Surg. 2011;37(3):446-53.

27. Campbell F, Green D. Monocular versus binocular visual acuity. Nature. 1965;208(5006):191-2.

28. Baker DH, Lygo FA, Meese TS, Georgeson MA. Binocular summation revisited: Beyond $\sqrt{ } 2$. Psychol Bull. 2018;144(11):1186.

29. Zheleznyak L, Sabesan R, Oh J-S, MacRae S, Yoon G. Modified monovision with spherical aberration to improve presbyopic through-focus visual performance. Investig Ophthalmol Vis Sci. 2013;54(5): $3157-65$.

30. Castro JJ, Soler M, Ortiz C, Jiménez JR, Anera RG. Binocular summation and visual function with induced anisocoria and monovision. Biomed Opt Express. 2016;7(10):4250-62. 